\title{
The Functional Ambulation: Standard Treatment versus Electrical Stimulation Therapy (FASTEST) trial for stroke: study design and protocol
}

This article was published in the following Dove Press journal:

Open Access Journal of Clinical Trials

14 February 2013

Number of times this article has been viewed

\section{Kari Dunning' \\ Michael O'Dell ${ }^{2}$ \\ Patricia Kluding ${ }^{3}$ \\ Samuel S Wu ${ }^{4}$ \\ Jody Feld ${ }^{5}$ \\ Jivan Ginosian ${ }^{6}$ \\ Keith McBride ${ }^{6}$}

'Department of Rehabilitation Sciences, College of Allied Health Sciences, University of Cincinnati, Cincinnati, $\mathrm{OH},{ }^{2}$ Department of Rehabilitation Medicine, New YorkPresbyterian Hospital/Weill Cornell Medical Center, New York, NY, ${ }^{3}$ Department of Physical Therapy and Rehabilitation Sciences, University of Kansas Medical Center, Kansas City, KS, ${ }^{4}$ Department of Biostatistics, University of Florida, Gainesville, $\mathrm{FL},{ }^{5}$ Department of Community and Family Medicine, Duke University School of Medicine, Durham, NC, ${ }^{6}$ Bioness Inc, Valencia, CA, USA
Correspondence: Kari Dunning Department of Rehabilitation Sciences, College of Allied Health Sciences, University of Cincinnati, 3202 Eden Ave, PO Box 670394, Cincinnati, $\mathrm{OH}$ 45267-0394, USA

$\mathrm{Tel}+\mathrm{I} 5 \mathrm{I} 35587477$

$\mathrm{Fax}+\mathrm{I} 5 \mid 35587474$

Email kari.dunning@uc.edu
Background: Surface electrical stimulation for foot drop (foot drop stimulation [FDS]) technology has greatly improved over the last decade, leading to increased use in the clinic environment and the community. Despite numerous studies suggesting the benefit of FDS among persons with stroke, there are no randomized controlled trials comparing FDS to standard of care (ankle foot orthosis [AFO]). The Functional Ambulation: Standard Treatment versus Electrical Stimulation Therapy (FASTEST) study is a single-blinded randomized controlled trial with the primary purpose of comparing FDS and AFO among persons with stroke conducted at eleven sites throughout the USA.

Methods: Persons $\geq 3$ months poststroke are randomized to wear either FDS or AFO for 30 weeks. After 30 weeks, AFO participants crossover to wear an FDS. All participants are followed for 42 weeks with repeated measures at baseline and Weeks 6, 12, 30, 36, and 42 . The primary analysis will compare gait speed between FDS and AFO at 30 weeks. Secondary outcomes span the International Classification of Functioning, Disability, and Health categories and include functional gait, balance, motor control, falls, and quality of life. Tertiary analyses will be performed using Weeks 36 and 42 time points.

Conclusion: This pivotal trial is the first longitudinal randomized controlled trial to compare FDS and AFO in persons with stroke. Further, the results will be the largest single contribution to date on the efficacy of FDS in people with stroke, providing a robust dataset with findings that can be extrapolated for use as guidelines to clinical practice.

Trial registration: clinicaltrials.gov NCT01138995.

Keywords: electrical stimulation, stroke, cerebrovascular accident, rehabilitation, foot drop stimulation, gait

\section{Introduction}

Each year 795,000 people experience a new or recurrent stroke in the USA, resulting in 7 million Americans living with stroke. ${ }^{1}$ Stroke is a leading cause of serious long-term disability in the USA. ${ }^{1}$ In fact, among stroke survivors $\geq 65$ years old, $50 \%$ had some hemiparesis and $30 \%$ were unable to walk without some assistance at 6 months poststroke. ${ }^{1}$ The ability to independently ambulate has been identified as a key determinant for survival. ${ }^{2}$ While $65 \%-85 \%$ of patients eventually ambulate independently after stroke, ${ }^{3-5}$ the majority of these independent ambulators still walk at speeds considered less than functional for community ambulation, ${ }^{6}$ thereby limiting their overall level of social participation. ${ }^{7}$ Therefore, interventions to effectively enhance gait have the potential to profoundly impact quality of life poststroke.

One of the most common and frustrating neurological deficits that result from stroke is foot drop. Foot drop is characterized by the inability to dorsiflex the foot sufficiently 
to lift the toes completely off the ground during the swing phase of walking. In addition, the foot on the hemiplegic leg often does not strike the ground in the normal way at initial contact (ie, heel strike). ${ }^{8}$ This may cause unstable and slow gait with increased risk of falls. Foot drop during the swing phase also increases walking effort because of compensations required to advance the leg. ${ }^{9}$ Additionally, weakness in the dorsiflexor muscles and/or spasticity in plantarflexor muscles has been associated with difficulty with other functional activities such as rising from a chair and has been linked to falls after a stroke. ${ }^{10-12}$ It has been reported that foot drop is a persistent form of long-term disability in 10\%-20\% of stroke survivors. ${ }^{13}$

Conventional, standard of care treatment for foot drop is an ankle foot orthosis (AFO), a plastic or metal support worn on the lower leg. AFOs provide ankle dorsiflexion support during the swing phase and improve knee stability during the early stance phase of gait. ${ }^{8,14}$ A recent systematic review of the effects of AFO in stroke had limited conclusive findings due to the lack of rigor and diversity of trials. ${ }^{15}$ The long-term effects of AFO use in persons with stroke have not been studied. Since the primary goal of the AFO is to provide support and positioning for weakened muscles and joints, this treatment has several potential limitations. For example, the typical AFO immobilizes the ankle, which may contribute to the development of contracture, ${ }^{16}$ and does not allow the person to use residual active foot movement. ${ }^{14}$ This potentially limits future recovery of ankle strength and function, promoting disuse atrophy in the hemiplegic lower leg. Solid (ie, unhinged) AFOs also hinder standing from a sitting position as they limit dorsiflexion, and initial foot position is a major determinant of the ability to stand up from a chair. ${ }^{17}$ A recent study of people with hemiplegia from stroke who had completed their rehabilitation found that although the use of AFOs improved their gait speed and balance, over $60 \%$ of the participants indicated that they prefer not to use their AFO because of aesthetics. ${ }^{18}$

Surface electrically induced dorsiflexion by means of a foot drop stimulator (FDS) has been reported in the clinical literature since the 1970s and has been in clinical use for many years. ${ }^{19}$ As an alternative to a traditional AFO, FDS can be used to activate the ankle muscles during walking. Traditional systems consisted of an external pulse generator with a pair of leads connected to surface hydrogel electrodes and another wire running to a heel switch placed in the patient's shoe. These design limitations have been linked to reports of decreased use. ${ }^{20}$ Technical advancement to FDS, such as wireless design, reproducible electrode placement via stimulation cuffs, and more sophisticated programming has allowed the devices to be used with greater ease in the clinic and the community. Studies suggest that among persons with stroke, FDS facilitates normal electromyographic activity and decreases flexor-extensor cocontraction; improves symmetry in stance and cycle time; improves knee and ankle kinematics and reduces energy cost; increases cortical activity; increases strength, mobility, and gait velocity; and improves functional ambulation such as walking over varied surfaces and negotiating turns, obstacles, and stairs. ${ }^{20-43}$

The benefits of FDS can be assessed in two ways: orthotic (while using the device) and therapeutic (carryover effects not using the device). The most recent systematic review addressing orthotic effects pooled data from six studies found a significant $(P<0.05)$ increase in gait speed of $0.13 \mathrm{~m} /$ second. $^{21}$ The most recent meta-analysis addressing therapeutic effects pooled data from three studies using a fixed effects model and found a mean difference in gait speed of $0.18 \mathrm{~m} / \mathrm{second}$ $(P<0.01) .{ }^{44}$ Although this cumulative evidence shows benefits of FDS for gait in persons with stroke, to date there have been no randomized controlled trials to compare the effects of FDS to standard of care AFO.

Patient compliance is a critical consideration. A few studies have reported on patients' preference between FDS and AFO. A recent study used a qualitative approach to examine orthotic preferences for people with stroke. Eight of the nine people interviewed preferred using FDS to the AFO, citing reasons of ankle mobility, walking ability, and comfort. ${ }^{45}$ After an 8 -week FDS intervention, 26 participants who formerly used an AFO significantly $(P<0.05)$ preferred the FDS due to comfort, appearance, and gait quality, distance, effort, and stability. ${ }^{34}$ In a similarly designed study among 15 participants with stroke, 13 felt more stable and 14 reported a more normal gait with the FDS compared to AFO. ${ }^{29}$ In a cross-sectional study, twelve of 14 participants preferred FDS to AFO, citing improved muscle movement, strength, and gait. ${ }^{24}$ Given the magnitude of foot drop in stroke and subsequent functional limitations, more rigorously designed trials are needed to assess the value of these devices in stroke rehabilitation.

The effect of FDS or an AFO on gait can be measured in several different ways, as illustrated in Figure 1. The immediate effect refers to changes in gait that occur when initially wearing the device. A training effect above and beyond the immediate effect may occur as the patient uses the AFO or FDS over time. The therapeutic effect refers to improvements in walking seen even without wearing an AFO or FDS and may result from changes in neural plasticity, peripheral 


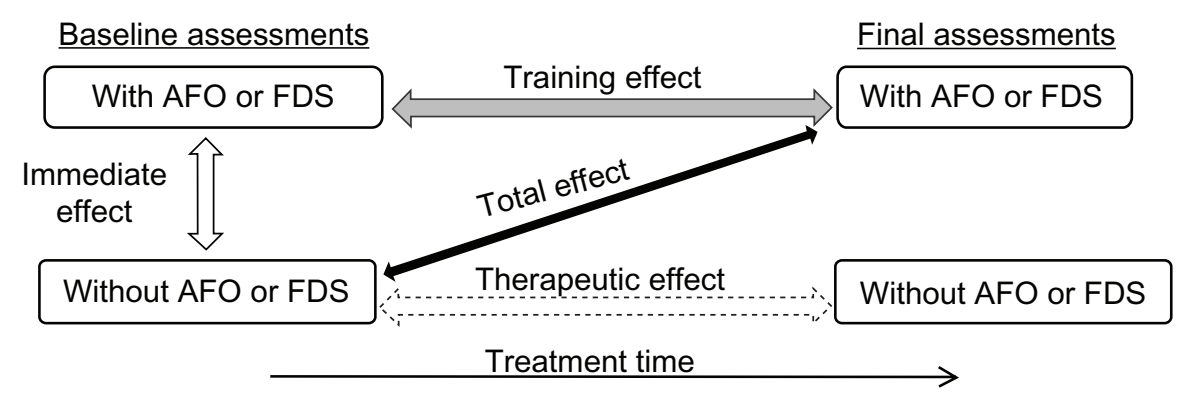

Figure I Illustration of possible comparison effects of device (FDS, AFO) on gait. Abbreviations: AFO, ankle foot orthosis; FDS, foot drop stimulation.

strength, cardiopulmonary system, or other systems. The total effect refers to the changes in gait that occur over time, and encompasses both the immediate and training effects.

The Functional Ambulation: Standard Treatment versus Electrical Stimulation Therapy (FASTEST) trial is designed to compare FDS and AFO for drop foot among people $>$ 3 months poststroke, with a gait speed $\leq 0.8 \mathrm{~m} /$ seconds. This is a multicenter, randomized controlled, single-blinded trial. The primary hypothesis is that after 30 weeks, participants randomized to the FDS group will demonstrate greater improvement in gait speed than participants randomized to the AFO group. This hypothesis was based on the anticipated total device effects, encompassing both the immediate and training effects. Other comparisons illustrated in Figure 1 will also be assessed. There are several secondary outcomes including gait function, balance, stroke-specific quality of life, and safety. Tertiary analyses will also be performed using Weeks 36 and 42 time points.

\section{Methods/design}

FASTEST is a multicenter, randomized controlled, partial crossover, single-blinded study in which persons $\geq 3$ months poststroke are followed for 42 weeks. Participants are randomized to either 30 weeks of wearing a surface FDS (original treatment group) or 30 weeks of AFO (original control group). After 30 weeks, the original control group crosses over to wear the FDS; whereas the original treatment group continues to wear the FDS. The FDS used in this protocol is the NESS L300 ${ }^{\circledR}$, manufactured by Bioness Inc (Valencia, CA, USA) who sponsored this study.

Participants are being recruited from eleven clinical sites across the USA (Figure 2). Each site has obtained local institutional review board approval except one that used Western Institutional Review Board (Olympia, WA, USA). Informed consent occurs during the first visit before any participant information is obtained and before any medical evaluations or baseline testing are performed. After consenting, the participant sign the Health Insurance Portability and Accountability Act authorization form and eligibility is confirmed (Table 1).

\section{Outcomes}

Repeated outcome measures are obtained using established, stroke-specific measures at Weeks 0, 6, 12, 30, 36, and 42 (Table 2). Outcomes are measured with and without the device (FDS or AFO) to assess several possible effects, as illustrated in Figure 1. An immediate effect refers to the change in gait that may occur when initially wearing the device: Visit 1 (V1) without device compared to V2 with device. A training effect may occur as the subject uses the orthotic over time: V2 with device compared to V8 with device. A therapeutic effect refers to the change that may occur in walking without the device: V1 without device compared to V8 without device. A total effect refers to the changes in gait that occur over time, and encompasses both the immediate and training effects: V1 without device compared with V8 with device.

Outcome testing is performed by licensed physical therapists who are blinded to the randomization group. To maintain blinding, a nonblinded research team member coordinates outcome testing and all subjects wear loose pants, a lower leg and shoe cover ("gaiter") on the involved lower extremity (to conceal the AFO or FDS cuff and pressure sensor), and an FDS control unit. Standardization of outcome measures is maximized across sites by providing test kits and outcome tester training followed by onsite competency testing.

\section{Primary outcome}

Gait speed measured by the $10 \mathrm{~m}$ walk test is the primary endpoint for this study. The $14 \mathrm{~m}$ course incorporates $2 \mathrm{~m}$ at beginning and end to allow for acceleration/deceleration. Both comfortable and "fast" gait speed are measured. The $10 \mathrm{~m}$ walk test is reliable in persons with stroke with intraclass correlation coefficient (ICC) values of $0.8-0.98,{ }^{46,47}$ and walking speed has been shown to be a predictor of 


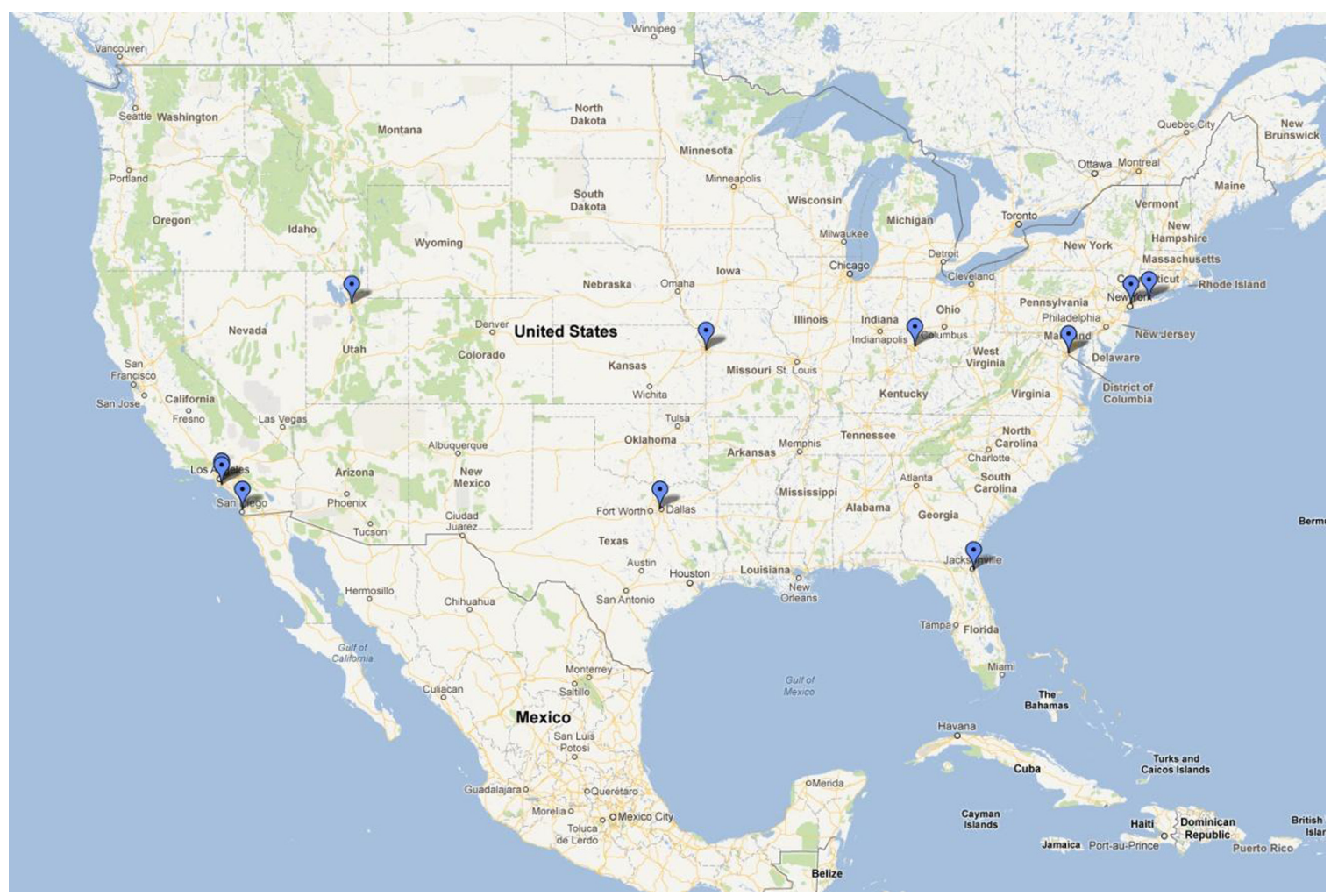

Figure 2 The eleven study sites include: University of Cincinnati, UC Health Drake Center; Weill Cornell Medical College; University of Kansas Medical Center; Long Beach Memorial Medical Center; University of Utah; Sharp Rehabilitation Center; Brooks Rehabilitation Hospital; UT Southwestern Medical Center; National Rehab Hospital; and St Charles Hospital and Rehabilitation.

community ambulation and functional status. ${ }^{649-52}$ During ambulation outcomes testing, the most commonly used assistive device ( $>50 \%$ time) at the time of assessment is utilized. The amount of assistance provided during each test is assigned using the Functional Ambulation Category, ${ }^{53-55}$ although no assistance is provided for the advancement of a paretic limb.

\section{Secondary outcomes}

The Timed Up and Go test is used to measure functional mobility. It is reliable and valid among persons with stroke. ${ }^{48,56}$ The 6-minute walk test is used to measure functional walking endurance..$^{57-59}$ This indoor walking course is on a level, noncarpeted surface measuring a length of $18 \mathrm{~m}$ with a $0.5 \mathrm{~m}$ turn at each end marked by a cone. One chair is positioned before the start line, one past the $18 \mathrm{~m}$ mark, and one to two chairs are staggered along the path at $6 \mathrm{~m}$ and $12 \mathrm{~m}$. This test is reliable in persons with stroke with ICC values of 0.97-0.99.48,60-62 The Berg Balance Scale is used to measure balance. It is a task performance test consisting of 14 items of increasing difficulty graded on a five-point ordinal scale of zero to four (zero = participant is unable to perform task; four $=$ participant is independent in performance of task). ${ }^{63}$ The Berg Balance Scale is highly reliable and valid and has been used in many previous studies among persons with stroke. ${ }^{64-66}$ The Stroke Impact Scale activities of daily living, instrumental activities of daily living, mobility, and participation subscales are used to measure quality of life. The Stroke Impact Scale and its subscales are valid, responsive, and robust. ${ }^{67-70}$ The Functional Reach Test is used to measure functional balance, ${ }^{71}$ and is reliable, valid and responsive. ${ }^{72-74}$ The Lower Extremity Fugl-Meyer motor subscale is used to measure motor recovery and impairment. The Fugl-Meyer has been reported to be an effective tool to quantify motor impairment in stroke survivors and to stratify severity of impairments, ${ }^{75}$ and is reliable with ICC values of $0.89-0.96 .^{76-78}$ Falls are obtained by self-report from participants and/or their caregivers retrospectively 6 months prior to baseline and at each study visit during the 30-week intervention period. Circumstances regarding each fall are collected, including any injury or medical attention received. A user satisfaction survey is completed at Week 12 (after completion of physical therapy sessions) and again at Week 30 in both groups. This twelve-item survey has a total score range from zero to 24 , with a higher number indicating greater satisfaction with the device. 
Table I Eligibility criteria

\begin{tabular}{|c|c|}
\hline Inclusion criteria & Exclusion criteria \\
\hline $\begin{array}{l}\text { - Ankle dorsiflexion range of motion } \geq \text { neutral when assessed } \\
\text { concurrently with test stimulation in a sitting and standing } \\
\text { position } \\
\text { - Adequate ankle and knee stability during gait at the time of } \\
\text { screening } \\
\text { - } \geq \text { I stroke of any etiology (eg, ischemic, hemorrhagic) } \\
\text { experienced } \geq 3 \text { months prior to study enrollment, as } \\
\text { confirmed by independent medical records (if available) } \\
\text { - Drop foot due to stroke sufficient to require use of an AFO } \\
\text { - Adequate cognition and communication abilities demonstrated } \\
\text { by either the participants scoring } \geq 24 \text { (out of a possible } 30 \text { ) } \\
\text { on the Mini Mental State Examination or having a competent } \\
\text { caregiver } \\
\text { - } \geq 18 \text { years } \\
\text { Able to safely walk } \geq 10 \text { m with a maximum of one person } \\
\text { assisting } \\
\text { - Self-selected (ie, comfortable) I0 m gait speed } \leq 0.80 \text { m/second } \\
\text { at the time of baseline assessment }\end{array}$ & 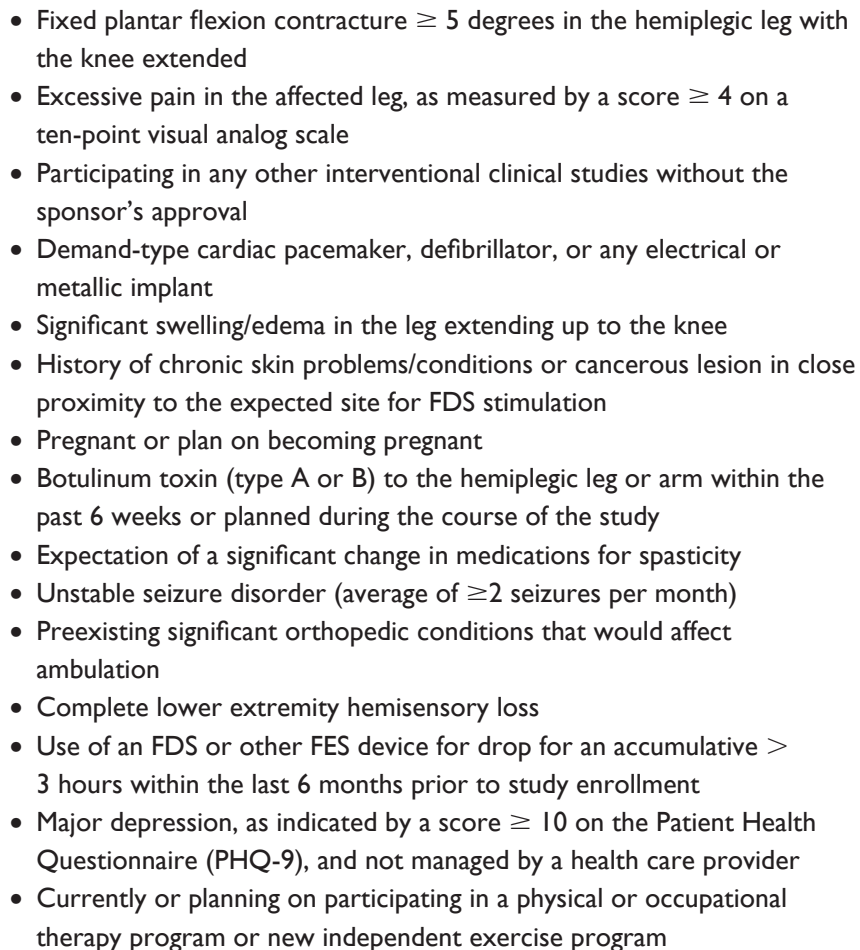 \\
\hline
\end{tabular}

Abbreviations: AFO, ankle foot orthosis; FDS, foot drop stimulation; FES, functional electrical stimulation.

A step activity monitor (StepWatch ${ }^{\mathrm{TM}}$; Orthocare Innovations, Oklahoma City, OK, USA) is worn for 7 days during Weeks 8 and 24 to quantify the amount of walking and to monitor compliance. Among the original control group, the step activity monitor is worn on both the nonparetic leg (to measure amount of walking) and the paretic leg (attached to the AFO to monitor compliance). Among the original treatment group, the step activity monitor is worn only on the nonparetic limb to measure amount of walking and the FDS is used to monitor compliance. The step activity monitor has been shown to be reliable, valid, and sensitive to changes in daily activity. ${ }^{79-82}$

\section{Randomization}

After baseline testing without the FDS/AFO, participants are randomly assigned by Bioness staff (JG) using an online program prepared by the study statistician (SW) in a process that is concealed by the site. Covariate adaptive randomization ${ }^{83,84}$ is used to ensure balanced allocation at each site for age, time poststroke, and known confounders ${ }^{85-88}$ within four subgroups: 3-6 months poststroke, $>6$ months poststroke, $<65$ years old, and $\geq 65$ years old or a Medicare beneficiary. Persons $>6$ months poststroke provide a more stable baseline "plateaued recovery state" and $\geq 65$ years old or a Medicare beneficiary addresses the largest single insurance provider covering durable medical equipment following stroke.

\section{Interventions}

All participants, regardless of group assignment, undergo an AFO evaluation prior to randomization by an orthotist and physical therapist trained on "best practice points." ${ }^{99}$ Although the intent of the study is to provide a comparison of the FDS with broadly-defined, standard-of-care "usual" AFO, a new AFO prescription is provided if indicated by the study team. The specific type of AFO (eg, solid ankle, hinged) prescribed for each participant is left to the discretion of the investigational staff. The orthotist and interventional physical therapist have undergone standardized training and onsite competency assessment.

\section{Physical therapy intervention}

During the first 6 weeks of the study, both groups receive eight sessions of physical therapy (twice a week for the first 2 weeks and once a week for the next 4 weeks). The original control group receives an additional 6 weeks (eight visits) of physical therapy after the 30 -week crossover when they receive their FDS. 
Table 2 Summary of outcome measures by time. Testing is performed with the subject using the device - foot drop stimulation or ankle foot orthosis - unless otherwise specified by "wo" (ie, without device)

\begin{tabular}{|c|c|c|c|c|c|c|c|c|c|c|c|}
\hline Assessment & $\begin{array}{l}\text { Baseline } \\
\text { (VI) }\end{array}$ & $\begin{array}{l}\text { V2 } \\
(\text { Wk 0) }\end{array}$ & $\begin{array}{l}\text { V3 } \\
(\text { Wk 6) }\end{array}$ & $\begin{array}{l}\text { V4 } \\
\text { (Wk I2) }\end{array}$ & Wk 16 & Wk 20 & Wk 24 & $\begin{array}{l}\text { V8 } \\
\text { (Wk 30) }\end{array}$ & $\begin{array}{l}\text { Crossover } \\
\text { visit' }\end{array}$ & $\begin{array}{l}\text { V9 } \\
\text { (Wk 36) }\end{array}$ & $\begin{array}{l}\text { VIO } \\
\text { (Wk 42) }\end{array}$ \\
\hline \multicolumn{12}{|l|}{ Primary outcome } \\
\hline $\begin{array}{l}10 \mathrm{~m} \text { walk test } \\
\text { (gait speed) }\end{array}$ & X (wo) & $x$ & $\mathrm{x}$ & $x$ & & & & $X(w, w o)$ & $x$ & $x$ & $x$ \\
\hline \multicolumn{12}{|l|}{ Secondary outcomes } \\
\hline Timed Up and Go test & X (wo) & $x$ & $x$ & $x$ & & & & $X(w, w o)$ & $x$ & $x$ & $x$ \\
\hline 6-minute walk test & $X($ wo $)$ & $x$ & $x$ & $x$ & & & & $X(w, w o)$ & $x$ & $x$ & $x$ \\
\hline Berg Balance Scale & $X$ (wo) & $x$ & $x$ & $x$ & & & & $X(w, w o)$ & $x$ & $x$ & $x$ \\
\hline Functional Reach Test & X (wo) & $x$ & $x$ & $x$ & & & & $X(w, w o)$ & $x$ & $x$ & $x$ \\
\hline Lower Extremity & $x$ & $x$ & $x$ & $x$ & & & & $x$ & $x$ & $x$ & $x$ \\
\hline \multicolumn{12}{|l|}{ Fugl-Meyer ${ }^{2}$} \\
\hline Stroke Impact Scale ${ }^{2}$ & $x$ & $x$ & $x$ & $x$ & & & & $x$ & $x$ & $x$ & $x$ \\
\hline Falls questionnaire ${ }^{3}$ & $x$ & $x$ & $x$ & $x$ & $x$ & $x$ & $x$ & $x$ & $x$ & $x$ & $x$ \\
\hline $\begin{array}{l}\text { Device satisfaction } \\
\text { questionnaire }^{4}\end{array}$ & & & & $x$ & & & & $x$ & & & $\mathrm{x}$ \\
\hline Step activity monitor & & & $x$ & & & & $x$ & & & & \\
\hline
\end{tabular}

Notes: 'Crossover visit tests only for the control group (starting with the crossover visit through to the end of study, the control group is tested using the FDS, ie, no longer tested with AFO); ${ }^{2}$ always tested without the device; ${ }^{3}$ also collected at each physical therapy visit; ${ }^{4}$ performed for FDS and AFO, depending on group assignment (for the original control group, questions address the FDS after crossover).

Abbreviations: AFO, ankle foot orthosis; FDS, foot drop stimulation; Wk, Week; wo, testing occurs without device (FDS or AFO); V, outcome testing visit.

Treatment time for the first therapy session is 60 minutes, the second session 45 minutes, and the remaining six sessions range from 30-45 minutes. Regardless of randomization group, the first two to four therapy visits focus on education for use of AFO or FDS, initial gait training with the prescribed device, and the development of an individualized 30-minute home exercise program. During the remaining sessions, therapy includes gait training with the FDS or AFO addressing the primary limitations to safe and independent ambulation in the home and community as determined by the physical therapist's initial and ongoing assessments. Each physical therapy session includes a skin assessment. In addition, participants receive group specific education and therapeutic intervention as outlined below.

\section{Original treatment group (FDS protocol)}

Standard of care Bioness clinical protocols for the initial fitting, follow-up, home use, and conditioning of the FDS are used..$^{90}$ Education on the use and maintenance of the FDS is provided throughout the eight therapy visits. For the first 3 weeks, treatment participants follow the standard conditioning protocol that involves gradually increasing walking with the FDS from 15 minutes each day to all day use. The Bioness skin care guidelines ${ }^{90}$ are reviewed and provided to the participant during the initial fitting. The first 3 weeks also involves using the FDS for cyclic stimulation ("training") when the participant is not walking. The goal of this training is to gradually strengthen and condition the muscles responsible for ankle dorsiflexion and eversion to avoid fatigue when using the FDS. Training is done 15 minutes two times daily for 1 week followed by 20 minutes two times daily over the next 2 weeks. An 8-hour break between training sessions minimizes fatigue and overuse.

\section{Original control group (AFO protocol)}

The control group is designed to maximize safety with the AFO and minimize bias (compared to the treatment group). Education on the use, care, and maintenance of the AFO is provided. An AFO wearing schedule is provided as needed (eg, new AFO). AFO instructions and skin care guidelines are provided and reviewed during the eight therapy visits. To reduce potential treatment bias, control participants receive surface sensory stimulation with a commercially available transcutaneous electrical nerve stimulation (TENS) device at each physical therapy visit, corresponding to the approximate time FDS is used in the treatment group. During the first week, participants receive 30 minutes of TENS at each physical therapy visit; during the next 2 weeks, they receive 30-45 minutes of TENS at each physical therapy visit. The TENS device is placed on the hemiplegic leg in a location that corresponds anatomically to the location where the treatment participants receive FDS stimulation. The TENS device is set to the lowest stimulation level that first yields a sensory response but no motor response. The settings for the TENS device include: continuous stimulation at a frequency 
of 100 pulses/second, duration of 200 microseconds, and amplitude setting to elicit sensory response only. After receiving the FDS at the Week 30 crossover visit, the control group follows the FDS protocol outlined above.

\section{Well visits}

Well visit follow-ups performed at Weeks 16, 20, and 24 include a falls questionnaire and skin assessment. These visits also ensure participants are using the prescribed devices safely and appropriately between the outcome measure visits.

\section{Sample size and power analysis}

Recent studies suggest a standard deviation of $0.20 \mathrm{~m} / \mathrm{second}$ for walking speed change from baseline to follow-up. ${ }^{25,26,41}$ The original plan was to enroll 176 eligible participants (resulting in 132 with an estimated 25\% drop out), allowing for $80 \%$ power to detect a clinically meaningful $(0.1 \mathrm{~m} /$ second $)^{67}$ difference in walking speed change between groups using a two-sample $t$-test with a two-sided 0.05 level. After the first planned interim analysis (September 2011), the enrollment goal was increased to 206. As a result of favorable trends in outcomes for participants with severe gait impairment ( $<0.4 \mathrm{~m} /$ second gait speed), the hypothesis was added that among participants in this subgroup, those randomized to the FDS group would demonstrate greater improvement in gait speed than those randomized to the AFO group. This gait impairment category ( $<0.4 \mathrm{~m} /$ second) was chosen based on well-accepted categories of community ambulation ${ }^{6}$ and previous research. ${ }^{91-94}$ This increase in sample size allowed for: (1) the addition of a primary hypothesis for a subgroup of persons with initially severe gait impairment, and (2) to reduce the risk of type II errors on several secondary outcomes.

\section{Statistical analysis}

This study will test the primary hypotheses by comparing changes in outcomes from baseline to 30 weeks between treatment and control groups. The primary hypothesis is that participants using FDS will increase gait speed more than participants using an AFO, as measured by the total effect. This hypothesis will be examined in the entire group of participants, and also in the subgroup of persons with severe gait impairment $(<0.4 \mathrm{~m} / \mathrm{second}$ gait speed $)$ at baseline. The following statistical approach will be taken to test the hypotheses.

First, demographic and baseline variables will be compared between the two randomly assigned groups using a $t$-test for comparison of means and chi-squared tests for comparison of proportions. Variables found to be significantly different between the groups will be used as covariates in the final analyses, in addition to prespecified covariates ( site and prestudy AFO use).

Second, the primary intention-to-treat analysis will be performed at the 0.05 level. The study-wide error rate will be controlled by applying Hochberg's step-up procedure to two tests: one for the entire group and the other for the severe subgroup. Each statistical test will be based on Fisher's combination of two $P$-values: one from before and the other from after the first interim look. Both $P$-values will be derived from a linear regression model with walking speed improvement from baseline to 30 weeks as the dependent variable and the treatment group as the independent variable, controlling for prespecified covariates and pretreatment intergroup differences. For participants who do not complete the 30 weeks evaluation, their outcomes will be predicted by a fitted model that will take into account participant dropout bias. ${ }^{95}$ More specifically, these three steps will be followed: (1) determine demographic and clinical variables that characterize differences between "completers" and "noncompleters;" (2) develop a model predicting outcomes for the "completers" using the significant independent variables from step one; (3) use the resulting model to predict outcomes for the "noncompleters," with the provision that for those who dropped out due to a related adverse event, their gait velocity changes will be imputed as the minimum of zero and the predicted changes.

If the above primary analysis is statistically significant, two subgroup analyses will be conducted using a one-sided twosample $t$-test at the 0.05 level: one for those who are $\geq 65$ years old or a Medicare beneficiary and the other for those who are $\geq 6$ months poststroke. In addition, sensitivity analyses will be performed by comparing results from the intentionto-treat analyses described above. Also, the chi-squared test will be applied to compare the two randomized groups in the number of participants who move into new classes of clinically meaningful functional ambulation; participants will be divided into three categories: (1) who progress higher, (2) who do not change, and (3) who regress in their ambulation class status, eg, "household ambulators" ( $<0.4 \mathrm{~m} /$ second), "limited community ambulators" (0.4-0.8 m/second), and "full community ambulators" ( $>0.8 \mathrm{~m} / \mathrm{second}{ }^{6}{ }^{6}$

Third, Wilcoxon rank-sum tests will be conducted to compare secondary outcomes between the two groups. For simplicity, the "completers" only will be analyzed and there will be no adjustment for covariates. However, the familywise 
error rate will be controlled at the 0.05 level for all secondary hypotheses testing based on Holm's step-down procedure, which rejects a hypothesis only if its $P$-value and each of the smaller $P$-values are less than their corresponding critical values.

In addition, a few tertiary analyses will be performed to compare the two randomized groups on trajectory of walking speed change and rate of falls, as well as a within-group test on whether the primary and secondary outcomes improved, diminished, or remained relatively stable from 30 weeks to 36 and 42 weeks.

\section{Data management and quality}

A secure web-based electronic data capture system (Rave ${ }^{\circledR}$; Medidata Solutions Worldwide, New York, NY, USA) is used for clinical data collection and management. Third-party monitors perform regular visits at each site to review and verify all study data in source documents.

\section{Study organization and management}

The sponsor (Bioness Inc) is responsible for implementation of this protocol at each investigational site and ensuring that all data are collected in compliance with study protocol. They implement protocol changes, train study personnel, and oversee clinical/regulatory aspects of the trial in accordance with International Conference on Harmonization/Good Clinical Practice Guidelines and by Code of Federal Regulations Title 21. The sponsor also developed and maintains the manual of procedures, data collection forms, and the electronic database (Rave). With coordination from an independent publications committee, the sponsor will review and provide written consent prior to independent publications, presentations, or public disclosures. The sponsor will have no direct involvement in statistical analysis.

\section{Discussion}

This FASTEST multicenter randomized controlled trial is designed to compare the effectiveness of FDS to an AFO for management of drop foot in people with subacute and chronic stroke. The scope of this study is unparalleled in FDS research including: number of participants, number of sites, number of outcome measures, and the breadth of those measures across the entire International Classification of Functioning, Disability, and Health. There have been other FDS studies incorporating some of the design elements used in FASTEST, but not comparing standard of care (AFO), randomized, blind, and with an adequately powered sample size. Further, this study conducted six repeated measures over a 42-week period, allowing for investigation of longitudinal response and change.

Due to recent technology improvements, the use of FDS is increasing in the clinic, home, and community. However, AFOs are standard of care typically covered by third-party payors who consider FDS investigational. Previous studies comparing FDS and AFO have design limitations (eg, withinsubject, cross-sectional designs). This is the first longitudinal, randomized controlled trial that compares FDS and AFO. Further, the trial will measure different aspects of change (immediate, training, therapeutic, and total as shown in Figure 1), thereby contributing significant information on the impact of FDS as compared to AFO not only as an orthotic but in its potential to facilitate therapeutic improvement in performance over time.

As stroke is a leading cause of disability in adults, evidenced-based intervention choices are critical to maximize potential in this large and growing patient population. Specifically, interventions that assist in restoring and improving walking ability can provide benefit beyond basic mobility and therefore the selection of a device or therapy targeting gait is critical as it can potentially influence overall quality of life. Even though foot drop has been identified as a major contributor of walking limitation in stroke, knowledge of the efficacy of devices is limited in the existing body of evidence. The results of this trial will provide a more comprehensive understanding of the impact of FDS and AFOs in the treatment of drop foot in persons with stroke and potentially serve to guide clinical decision making. The summation of the findings may serve as the definitive manuscript to date for the overall performance of FDS in select patients with stroke and its appropriate position in the rehabilitation continuum.

\section{Acknowledgments}

The authors thank the site principal investigators, medical directors, and study coordinators: Brett Kissela, Johnny Wilkerson, and Lynn Sprafka (University of Cincinnati, UC Health Drake Center, Cincinnati, OH); Laura DiStasi (Weill Cornell Medical Center, New York, NY); Linda Ladesich, Mamatha Pasnoor, and Rupali Singh (University of Kansas Medical Center, Kansas City, KS); Steven R Edgley and Mary S Annis Pautler (University of Utah, School of Medicine, Salt Lake City, UT); Kathy Brady and Alexander Dromerick (National Rehabilitation Hospital, Washington, DC); Trevor Paris, Brooke Hoisington, and Molly Dunn (Brooks Center for Rehabilitation Studies, Jacksonville, FL); Jerome Stenehjem and Sara Settle (Sharp Rehabilitation Center, San Diego, CA); John Thottakara and Cindy Dolezal 
(University of Texas Southwestern, Dallas, TX); Jun Zhang and Mike Apostoli (St Charles Hospital and Rehabilitation, Port Jefferson, NY); Ziyad Ayyoub and Bob Boucher (Rancho Los Amigos National Rehabilitation Center, Downey, CA); and Diemha Hoang and Wendy Lazouras (Long Beach Memorial Medical Center, Long Beach, CA). The authors also wish to thank the medical safety monitor: Richard Zorowitz (Johns Hopkins, Bay View Medical Center, Baltimore, MD). This clinical trial was funded by Bioness Inc.

\section{Disclosure}

J Ginosian, J Feld, and K McBride were employed by Bioness Inc during the study period; $\mathrm{S}$ Wu was paid as a statistical consultant by Bioness Inc. All other authors report no conflicts of interest in this work.

\section{References}

1. Roger VL, Go AS, Lloyd-Jones DM, et al. Heart disease and stroke statistics - 2012 update: a report from the American Heart Association. Circulation. 2012;125(1):e2-e220.

2. Chiu HT, Wang YH, Jeng JS, Chen BB, Pan SL. Effect of functional status on survival in patients with stroke: is independent ambulation a key determinant? Arch Phys Med Rehabil. 2012;93(3): 527-531.

3. Friedman PJ. Gait recovery after hemiplegic stroke. Int Disabil Stud. 1990;12(3):119-122.

4. Skilbeck CE, Wade DT, Hewer RL, Wood VA. Recovery after stroke. J Neurol Neurosurg Psychiatry. 1983;46(1):5-8.

5. Wade DT, Wood VA, Heller A, Maggs J, Langton Hewer R. Walking after stroke. Measurement and recovery over the first 3 months. Scand $J$ Rehabil Med. 1987;19(1):25-30.

6. Perry J, Garrett M, Gronley JK, Mulroy SJ. Classification of walking handicap in the stroke population. Stroke. 1995;26(6):982-989.

7. Hill K, Ellis P, Bernhardt J, Maggs P, Hull S. Balance and mobility outcomes for stroke patients: a comprehensive audit. Aust J Physiother. 1997;43(3):173-180.

8. Fatone S, Gard SA, Malas BS. Effect of ankle-foot orthosis alignment and foot-plate length on the gait of adults with poststroke hemiplegia. Arch Phys Med Rehabil. 2009;90(5):810-818.

9. Franceschini M, Massucci M, Ferrari L, Agosti M, Paroli C. Effects of an ankle-foot orthosis on spatiotemporal parameters and energy cost of hemiparetic gait. Clin Rehabil. 2003;17(4):368-372.

10. Cheng PT, Chen CL, Wang CM, Hong WH. Leg muscle activation patterns of sit-to-stand movement in stroke patients. Am J Phys Med Rehabil. 2004;83(1):10-16.

11. Ng SS, Hui-Chan CW. Contribution of ankle dorsiflexor strength to walking endurance in people with spastic hemiplegia after stroke. Arch Phys Med Rehabil. 2012;93(6):1046-1051.

12. Lin PY, Yang YR, Cheng SJ, Wang RY. The relation between ankle impairments and gait velocity and symmetry in people with stroke. Arch Phys Med Rehabil. 2006;87(4):562-568.

13. Lyons GM, Sinkjaer T, Burridge JH, Wilcox DJ. A review of portable FES-based neural orthoses for the correction of drop foot. IEEE Trans Neural Syst Rehabil Eng. 2002;10(4):260-279.

14. Mulroy SJ, Eberly VJ, Gronely JK, Weiss W, Newsam CJ. Effect of AFO design on walking after stroke: impact of ankle plantar flexion contracture. Prosthet Orthot Int. 2010;34(3):277-292.

15. Guerra Padilla M, Molina Rueda F, Alguacil Diego IM. Effect of ankle-foot orthosis in postural control after stroke: a systematic review. Neurologia. December 15, 2011. [Epub ahead of print.]
16. Given JD, Dewald JP, Rymer WZ. Joint dependent passive stiffness in paretic and contralateral limbs of spastic patients with hemiparetic stroke. J Neurol Neurosurg Psychiatry. 1995;59(3):271-279.

17. Janssen WG, Bussmann HB, Stam HJ. Determinants of the sit-to-stand movement: a review. Phys Ther. 2002;82(9):866-879.

18. Dogan A, Mengulluoglu M, Ozgirgin N. Evaluation of the effect of ankle-foot orthosis use on balance and mobility in hemiparetic stroke patients. Disabil Rehabil. 2011;33(15-16):1433-1439.

19. Metz WD. Bioengineering: "drop foot" corrected by electrical stimulation. Science. 1972;176(4037):897-900.

20. Taylor PN, Burridge JH, Dunkerley AL, et al. Patients' perceptions of the Odstock dropped foot stimulator (ODFS). Clin Rehabil. 1999; 13(5):439-446.

21. Kottink AI, Oostendorp LJ, Buurke JH, Nene AV, Hermens HJ, IJzerman MJ. The orthotic effect of functional electrical stimulation on the improvement of walking in stroke patients with a dropped foot: a systematic review. Artif Organs. 2004;28(6):577-586.

22. Liberson WT, Holmquest HJ, Scot D, Dow M. Functional electrotherapy: stimulation of the peroneal nerve synchronized with the swing phase of the gait of hemiplegic patients. Arch Phys Med Rehabil. 1961;42:101-105.

23. Burridge J, Taylor P, Hagan S, Swain I. Experience of clinical use of the Odstock dropped foot stimulator. Artif Organs. 1997;21(3):254-260.

24. Sheffler LR, Hennessey MT, Naples GG, Chae J. Peroneal nerve stimulation versus an ankle foot orthosis for correction of footdrop in stroke: impact on functional ambulation. Neurorehabil Neural Repair. 2006;20(3):355-360.

25. Hausdorff JM, Ring H. The effect of the Ness L300 neuroprosthesis on gait stability and symmetry [abstract]. J Neurol Phys Ther. 2006; 30(4):198.

26. Hausdorff JM, Ring H. The effect of a new lower-limb neuroprosthesis on physical and social functioning [abstract]. J Neurol Phys Ther. 2006; 30(4):217.

27. Sheffler LR, Hennessey MT, Naples GG, Chae J. Improvement in functional ambulation as a therapeutic effect of peroneal nerve stimulation in hemiplegia: two case reports. Neurorehabil Neural Repair. 2007;21(4):366-369.

28. Laufer Y, Ring H, Sprecher E, Hausdorff JM. Gait in individuals with chronic hemiparesis: one-year follow-up of the effects of a neuroprosthesis that ameliorates foot drop. J Neurol Phys Ther. 2009;33(2): $104-110$.

29. Ring H, Treger I, Gruendlinger L, Hausdorff JM. Neuroprosthesis for footdrop compared with an ankle-foot orthosis: effects on postural control during walking. J Stroke Cerebrovasc Dis. 2009;18(1):41-47.

30. Stein RB, Chong S, Everaert DG, et al. A multicenter trial of a footdrop stimulator controlled by a tilt sensor. Neurorehabil Neural Repair. 2006; 20(3):371-379.

31. Stein RB, Everaert DG, Thompson AK, et al. Long-term therapeutic and orthotic effects of a foot drop stimulator on walking performance in progressive and nonprogressive neurological disorders. Neurorehabil Neural Repair. 2010;24(2):152-167.

32. Dunning K, Black K, Harrison A, McBride K, Israel S. Neuroprosthesis peroneal functional electrical stimulation in the acute inpatient rehabilitation setting: a case series. Phys Ther. 2009;89(5): 499-506.

33. Israel S, Kotowski S, Talbott N, Fisher K, Dunning K. The therapeutic effect of outpatient use of a peroneal nerve functional electrical stimulation neuroprosthesis in people with stroke: a case series. Top Stroke Rehabil. 2011;18(6):738-745.

34. van Swigchem R, Vloothuis J, den Boer J, Weerdesteyn V, Geurts AC. Is transcutaneous peroneal stimulation beneficial to patients with chronic stroke using an ankle-foot orthosis? A within-subjects study of patients' satisfaction, walking speed and physical activity level. J Rehabil Med. 2010;42(2):117-121.

35. Everaert DG, Thompson AK, Chong SL, Stein RB. Does functional electrical stimulation for foot drop strengthen corticospinal connections? Neurorehabil Neural Repair. 2010;24(2):168-177. 
36. Sabut SK, Sikdar C, Mondal R, Kumar R, Mahadevappa M. Restoration of gait and motor recovery by functional electrical stimulation therapy in persons with stroke. Disabil Rehabil. 2010;32(19): 1594-1603.

37. Sabut SK, Lenka PK, Kumar R, Mahadevappa M. Effect of functional electrical stimulation on the effort and walking speed, surface electromyography activity, and metabolic responses in stroke subjects. J Electromyogr Kinesiol. 2010;20(6):1170-1177.

38. Sabut SK, Sikdar C, Kumar R, Mahadevappa M. Functional electrical stimulation of dorsiflexor muscle: effects on dorsiflexor strength, plantarflexor spasticity, and motor recovery in stroke patients. NeuroRehabilitation. 2011;29(4):393-400.

39. Taylor PN, Burridge JH, Dunkerley AL, et al. Clinical use of the Odstock dropped foot stimulator: its effect on the speed and effort of walking. Arch Phys Med Rehabil. 1999;80(12):1577-1583.

40. Taylor P, Burridge J, Dunkerley A, et al. Clinical audit of 5 years provision of the Odstock dropped foot stimulator. Artif Organs. 1999;23(5): $440-442$.

41. Alon G, Hausdorff M, Ring H. One year follow-up of patients who are using the NESS L300 neuroprosthesis: effects on gait performance [abstract]. Stroke. 2008;39(2):549.

42. Hausdorff JM, Ring H. Effects of a new radio frequency-controlled neuroprosthesis on gait symmetry and rhythmicity in patients with chronic hemiparesis. Am J Phys Med Rehabil. 2008;87(1): 4-13.

43. Embrey DG, Holtz SL, Alon G, Brandsma BA, McCoy SW. Functional electrical stimulation to dorsiflexors and plantar flexors during gait to improve walking in adults with chronic hemiplegia. Arch Phys Med Rehabil. 2010;91(5):687-696.

44. Robbins SM, Houghton PE, Woodbury MG, Brown JL. The therapeutic effect of functional and transcutaneous electric stimulation on improving gait speed in stroke patients: a meta-analysis. Arch Phys Med Rehabil. 2006;87(6):853-859.

45. Bulley C, Shiels J, Wilkie K, Salisbury L. User experiences, preferences and choices relating to functional electrical stimulation and ankle foot orthoses for foot-drop after stroke. Physiotherapy. 2011;97(3): 226-233.

46. Fulk GD, Echternach JL. Test-retest reliability and minimal detectable change of gait speed in individuals undergoing rehabilitation after stroke. J Neurol Phys Ther. 2008;32(1):8-13.

47. Evans MD, Goldie PA, Hill KD. Systematic and random error in repeated measurements of temporal and distance parameters of gait after stroke. Arch Phys Med Rehabil. 1997;78(7):725-729.

48. Flansbjer UB, Holmback AM, Downham D, Patten C, Lexell J. Reliability of gait performance tests in men and women with hemiparesis after stroke. $J$ Rehabil Med. 2005;37(2):75-82.

49. Maeda A, Yuasa T, Nakamura K, Higuchi S, Motohashi Y. Physical performance tests after stroke: reliability and validity. Am J Phys Med Rehabil. 2000;79(6):519-525.

50. Lord SE, McPherson K, McNaughton HK, Rochester L, Weatherall M. Community ambulation after stroke: how important and obtainable is it and what measures appear predictive? Arch Phys Med Rehabil. 2004; 85(2):234-239.

51. Wagenaar RC, Beek WJ. Hemiplegic gait: a kinematic analysis using walking speed as a basis. $J$ Biomech. 1992;25(9):1007-1015.

52. Nadeau S, Arsenault AB, Gravel D, Bourbonnais D. Analysis of the clinical factors determining natural and maximal gait speeds in adults with a stroke. Am J Phys Med Rehabil. 1999;78(2):123-130.

53. Holden MK, Gill KM, Magliozzi MR. Gait assessment for neurologically impaired patients. Standards for outcome assessment. Phys Ther. 1986;66(10):1530-1539.

54. Viosca E, Martinez JL, Almagro PL, Gracia A, Gonzalez C. Proposal and validation of a new functional ambulation classification scale for clinical use. Arch Phys Med Rehabil. 2005;86(6):1234-1238.

55. Kollen B, Kwakkel G, Lindeman E. Time dependency of walking classification in stroke. Phys Ther. 2006;86(5):618-625.
56. Ng SS, Hui-Chan CW. The Timed Up and Go test: its reliability and association with lower-limb impairments and locomotor capacities in people with chronic stroke. Arch Phys Med Rehabil. 2005;86(8): 1641-1647.

57. Salbach NM, Mayo NE, Wood-Dauphinee S, Hanley JA, Richards CL, Cote R. A task-orientated intervention enhances walking distance and speed in the first year post stroke: a randomized controlled trial. Clin Rehabil. 2004;18(5):509-519.

58. Pohl PS, Duncan PW, Perera S, et al. Influence of stroke-related impairments on performance in 6-minute walk test. J Rehabil Res Dev. 2002;39(4):439-444.

59. Muren MA, Hutler M, Hooper J. Functional capacity and health-related quality of life in individuals post stroke. Top Stroke Rehabil. 2008;15(1): $51-58$.

60. Eng JJ, Dawson AS, Chu KS. Submaximal exercise in persons with stroke: test-retest reliability and concurrent validity with maximal oxygen consumption. Arch Phys Med Rehabil. 2004;85(1):113-118.

61. Fulk GD, Echternach JL, Nof L, O'Sullivan S. Clinometric properties of the six-minute walk test in individuals undergoing rehabilitation poststroke. Physiother Theory Pract. 2008;24(3):195-204.

62. Liu J, Drutz C, Kumar R, et al. Use of the six-minute walk test poststroke: is there a practice effect? Arch Phys Med Rehabil. 2008;89(9): 1686-1692.

63. Berg K, Wood-Dauphinee S, Williams JI, Gayton D. Measuring balance in the elderly: preliminary development of an instrument. Physiother Can. 1989;41(6):304-311.

64. Berg KO, Maki BE, Williams JI, Holliday PJ, Wood-Dauphinee SL. Clinical and laboratory measures of postural balance in an elderly population. Arch Phys Med Rehabil. 1992;73(11):1073-1080.

65. Blum L, Korner-Bitensky N. Usefulness of the Berg Balance Scale in stroke rehabilitation: a systematic review. Phys Ther. 2008;88(5): 559-566.

66. Juneja G, Czyrny JJ, Linn RT. Admission balance and outcomes of patients admitted for acute inpatient rehabilitation. Am J Phys Med Rehabil. 1998;77(5):388-393.

67. Schmid A, Duncan PW, Studenski S, et al. Improvements in speedbased gait classifications are meaningful. Stroke. 2007;38(7): 2096-2100

68. Duncan PW, Bode RK, Min Lai S, Perera S. Rasch analysis of a new stroke-specific outcome scale: the Stroke Impact Scale. Arch Phys Med Rehabil. 2003;84(7):950-963.

69. Duncan PW, Lai SM, Bode RK, Perera S, DeRosa J. Stroke Impact Scale-16: a brief assessment of physical function. Neurology. 2003;60(2):291-296.

70. Duncan PW, Wallace D, Lai SM, Johnson D, Embretson S, Laster LJ. The stroke impact scale version 2.0. Evaluation of reliability, validity, and sensitivity to change. Stroke. 1999;30(10):2131-2140.

71. Duncan PW, Weiner DK, Chandler J, Studenski S. Functional reach: a new clinical measure of balance. J Gerontol. 1990;45(6):M192-M197.

72. Weiner D, Bongiorni DR, Studenski SA, Duncan PW, Kochersberger GG. Does functional reach improve with rehabilitation? Arch Phys Med Rehabil. 1993;74(8):796-800.

73. Duncan PW, Studenski S, Chandler J, Prescott B. Functional reach: predictive validity in a sample of elderly male veterans. J Gerontol Med Sci. 1992;47(3):M93-M98.

74. Tyson SF, DeSouza LH. Reliability and validity of functional balance tests post stroke. Clin Rehabil. 2004;18(8):916-923.

75. Gladstone DJ, Danells CJ, Black SE. The Fugl-Meyer assessment of motor recovery after stroke: a critical review of its measurement properties. Neurorehabil Neural Repair. 2002;16(3):232-240.

76. Duncan PW, Propst M, Nelson SG. Reliability of the Fugl-Meyer assessment of sensorimotor recovery following cerebrovascular accident. Phys Ther. 1983;63(10):1606-1610.

77. Sanford J, Moreland J, Swanson LR, Stratford PW, Gowland C. Reliability of the Fugl-Meyer assessment for testing motor performance in patients following stroke. Phys Ther. 1993;73(7):447-454. 
78. Hsueh IP, Hsu MJ, Sheu CF, Lee S, Hsieh CL, Lin JH. Psychometric comparisons of 2 versions of the Fugl-Meyer Motor Scale and 2 versions of the stroke rehabilitation assessment of movement. Neurorehabil Neural Repair. 2008;22(6):737-744.

79. Macko RF, Haeuber E, Shaughnessy M, et al. Microprocessor-based ambulatory activity monitoring in stroke patients. Med Sci Sports Exerc. 2002;34(3):394-399.

80. Haeuber E, Shaughnessy M, Forrester LW, Coleman KL, Macko RF. Accelerometer monitoring of home- and community-based ambulatory activity after stroke. Arch Phys Med Rehabil. 2004;85(12): 1997-2001.

81. Resnick B, Nahm ES, Orwig D, Zimmerman SS, Magaziner J. Measurement of activity in older adults: reliability and validity of the step activity monitor. J Nurs Meas. 2001;9(3):275-290.

82. Coleman KL, Smith DG, Boone DA, Joseph AW, del Aguila MA. Step activity monitor: long-term, continuous recording of ambulatory function. J Rehabil Res Dev. 1999;36(1):8-18.

83. Pocock SJ, Simon R. Sequential treatment assignment with balancing for prognostic factors in the controlled clinical trial. Biometrics. 1975;31(1):103-115.

84. Rosenberger WF, Lachin JM. Randomization in Clinical Trials: Theory and Practice. New York, NY: John Wiley and Sons; 2002.

85. Craig LE, Wu O, Bernhardt J, Langhorne P. Predictors of poststroke mobility: systematic review. Int J Stroke. 2011;6(4):321-327.

86. Kwakkel G, Kollen B, Twisk J. Impact of time on improvement of outcome after stroke. Stroke. 2006;37(9):2348-2353.

87. Duncan PW, Goldstein LB, Horner RD, Landsman PB, Samsa GP, Matchar DB. Similar motor recovery of upper and lower extremities after stroke. Stroke. 1994;25(6):1181-1188.
88. Veerbeek JM, Koolstra M, Ket JC, van Wegen EE, Kwakkel G. Effects of augmented exercise therapy on outcome of gait and gait-related activities in the first 6 months after stroke: a meta-analysis. Stroke. 2011;42(11):3311-3315.

89. Condie E, Campbell J, Martina J, editors. Report of a Consensus Conference on the Orthotic Management of Stroke Patients; September 21-26, 2003; Ellecom, The Netherlands. Copenhagen: International Society for Prosthetics and Orthotics; 2004.

90. Bioness Inc. NESS L300 ${ }^{\circledR}$ Clinician's Guide. Valencia, CA: Bioness Inc; 2006. Available from: http://www.bioness.com/Documents/ L300HCP/L300_Clinician_Guide.pdf. Accessed December 3, 2012.

91. Duncan PW, Sullivan KJ, Behrman AL, et al; LEAPS Investigative Team. Body-weight-supported treadmill rehabilitation after stroke. N Engl J Med. 2011;364(21):2026-2036.

92. Schmid A, Duncan PW, Studenski S, et al. Improvements in speedbased gait classifications are meaningful. Stroke. 2007;38(7): 2096-2100.

93. Plummer P, Behrman AL, Duncan PW, et al. Effects of stroke severity and training duration on locomotor recovery after stroke: a pilot study. Neurorehabil Neural Repair. 2007;21(2):137-151.

94. Tilson JK, Wu SS, Cen SY, et al. Characterizing and identifying risk for falls in the LEAPS study: a randomized clinical trial of interventions to improve walking poststroke. Stroke. 2012;43(2):446-452.

95. National Research Council. The Prevention and Treatment of Missing Data in Clinical Trials. Washington, DC: National Academies Press; 2010.
Open Access Journal of Clinical Trials

\section{Publish your work in this journal}

The Open Access Journal of Clinical Trials is an international, peerreviewed, open access journal publishing original research, reports, editorials, reviews and commentaries on all aspects of clinical trial design, management, legal, ethical and regulatory issues, case record form design, data collection, quality assurance and data auditing

\section{Dovepress}

methodologies. The manuscript management system is completely online and includes a very quick and fair peer-review system, which is all easy to use. Visit http://www.dovepress.com/testimonials.php to read real quotes from published authors. 